\title{
Modelling the Duration of Multihop Paths in Mobile Ad Hoc Networks
}

\author{
Gonçalo Jacinto, Nelson Antunes and António Pacheco
}

\begin{abstract}
Mobile ad hoc networks are characterized by having nodes that are cooperative and communicate without any kind of infrastructure. The mobility and multihop capability of these networks leads the network topology to change rapidly and unpredictably; this aspect must be incorporated in effective models to describe the dynamics of multihop paths.

When modeling the duration of multihop paths, a great part of the literature assumes that the links of multihop paths behave independently. This simplifies the modeling and reduces the complexity of computations. However, each link shares a common node with each of its neighbor links, turning the independent link assumption generally not valid. In this paper, we use a piecewise deterministic Markov model that characterizes the random behaviour of a multihop path not assuming independent links. We obtain the mean path duration of multihop paths and compare the results for the used model with the ones obtained by assuming independent links. Numerical results illustrate that independent link approximation results underestimate the mean path duration, with the most significant differences being observed with low node mobility and higher path durations.
\end{abstract}

\section{Introduction}

The demand for wireless communications is experiencing a steady growth. In this respect, the integration of Mobile Ad Hoc Networks (MANETs) multihop capa-

Gonçalo Jacinto

DMAT/CIMA-UE and ECT of University of Évora,

Nelson Antunes

FCT of University of Algarve and CEMAT, e-mail: nantunes@ualg.pt e-mail: gjcj@uevora.pt

António Pacheco

CEMAT and Department of Mathematics, Instituto Superior Técnico - TULisbon, e-mail: apacheco@math.ist.utl.pt 
bility into wireless networks is one of the most promising architectural upgrade to envisage area coverage without significant additional infrastructure cost. The rapid deployment and low configuration profile of MANETs make them suitable to be used in emergency and military scenarios, as well in sensor networks and vehicular networks, among others.

In MANETs the nodes can dynamically form a network in a self-organizing manner without the need of an existing fixed infrastructure. Nodes are expected to act cooperatively in order to route traffic and to allow the network to adapt to the highly dynamic status of its links and node mobility patterns. In view of the limited transmission range of nodes, when the source and destination nodes are at a distance greater than the transmission range, the communication between them is made by a multiple hop path, using the neighbor nodes to forward the traffic towards the destination node.

Once a multihop path is active, the mobility of nodes causes the frequent failure of the path and activation of a new link. Thus, node mobility affects the performance of a MANET (cf, e.g., [7] and [2]). Therefore, the development of models integrating mobility and the connectivity demands of MANETs are essential to characterize the reliability of these networks. The functionality of the network depends on the reliability of communications paths and these are dependent on the stability of the links along the multihop path and their dependence structure, with the state of the links limited by power constraints and channel effects.

Few studies in the literature address the reliability of multihop paths, with an exact analysis of this issue seeming to be limited to [1]. Moreover, most analytical studies that focus on link stability extend the analysis for multihop paths assuming that the links of a multihop path behave independently of each other (e.g., [4, 7, 8]).

One of the earliest analysis that includes mobility was done by McDonald and Znati [7], who addressed link and path availability assuming independent links and that nodes move according to a variation of the random walk mobility model. Xu et $a l$. [8] used a Markov chain mobility model to derive several path metrics assuming that links behave independently. Han et al. [4] proved that when the link count is large, the distribution of path duration converges to an exponential distribution. $\mathrm{La}$ and Han [6] relaxed the independence assumption for the links in [4] requiring that the dependence between links goes away asymptotically with increasing link count. In addition, Bai et al. [2] investigated path durations under different mobility models and routing protocols based on simulations.

An effective piecewise deterministic Markov model that describes the random behaviour of a multihop path in a MANET is proposed in [1]. We will use this model, which assumes that the links of a multihop path do not behave independently, to compute the mean path duration and compare the results obtained from this model with those obtained assuming that links behave independently.

The path is characterized through a Piecewise Deterministic Markov Processes (PDMP, see [3]) where, for simplicity, the mobility of each node along the path is given by the random walk mobility model. A PDMP is a Markov process that follows deterministic trajectories between random jumps, which occur either spontaneously, in a Poisson-like fashion, or when the process hits the boundary of its 
state space. The usage of a PDMP to model a multihop path arises naturally, since PDMPs are a mix of deterministic motion and random events, just like the multihop path dynamics.

The mean path duration is obtained as the unique solution of a set of ordinary differential equations (ODEs), that calculated by a recursive method allow to obtain numerical solutions of the mean path duration. The numerical results obtained for the mean path duration are compared with those obtained when assuming independent links. We show that the independent links assumption can lead to a large underestimation of the mean path duration, especially in scenarios where there is a small number of links and a low node mobility, which originates larger mean path durations.

The paper is organized as follows. Section 2 describes the multihop path model. Section 3 presents a recursive method for the computation of the mean path duration. Numerical results are presented in Section 4. Finally, Section 5 concludes the paper.

\section{Multihop Path Model}

We assume that a multihop path is set-up (or already active) at time 0 with $N-1$ links and extends from node 1 along nodes $2,3, \ldots$, until it reaches terminal node $N$. We consider a transmission range $R$ equal for all nodes in the multihop path and, given two consecutive nodes in the path, $i-1$ and $i$, with locations in the plane $l^{i-1}$ and $l^{i}$, respectively, they can communicate if $\left\|l^{i-1}-l^{i}\right\|<R$.

Each node in the path moves across the plane independently of other nodes according to a variation of the random walk mobility model. In this model it is assumed that a node alternates between two phases: pause (0) and move (1). If at a transition instant a node goes into phase $i$, the amount of time it stays in phase $i$ is drawn independently of the past according to a continuous distribution function $F_{i}$ with support on the set $\mathbb{R}^{+}$. We assume that the hazard rate function of $F_{i}$, denoted by $\lambda_{i}(t)=\mathrm{d} F_{i}(t) /\left(1-F_{i}(t)\right)$, is bounded on the positive reals.

Denote by $p^{j}$ the phase of node $j$ and by $m^{j}$ its mobility vector if $p^{j}=1$ (i.e the node is in the move phase). When the phase of node $j$ changes to move, the node picks a mobility vector according to a distribution function $F_{M}$ on an open set $S_{M}$. Choosing a mobility vector $m$ corresponds to choosing independently a direction $\theta$ and a velocity $v$ through $m=(v \cos \theta, v \sin \theta)$. The node travels from the current location in the direction and with the velocity drawn for the mobility vector during the entire phase duration, with distribution $F_{1}$. Once this time expires, independently of the past, the node pauses for a random time period with distribution $F_{0}$ before starting to move again.

To obtain the duration of a link between two nodes, we need to define the relative location and relative mobility vector of node $i$ with respect to node $i-1$, which are given, respectively, by $l_{r}^{i}=l^{i}-l^{i-1}$ and $m_{r}^{i}=m^{i}-m^{i-1}$, where $m^{i}$ (resp. $m^{i-1}$ ) is omitted in the expression if $p^{i}=0$ (resp. $p^{i-1}=0$ ), and if both nodes are in pause phases $m_{r}^{i}=\mathbf{0}$ with $\mathbf{0}=(0,0)$. Then, the duration of the link $i$, is given by 
$d_{\text {link }}\left(l_{r}^{i}, m_{r}^{i}\right)=Z\left(l_{r}^{i}, m_{r}^{i}\right) /\left\|m_{r}^{i}\right\|$, for $m_{r}^{i} \neq \mathbf{0}$, where $Z\left(l_{r}^{i}, m_{r}^{i}\right)$ denotes the distance that node $i$ needs to travel to move out of the range of node $i-1$.

The multihop path model is characterized by a vector of phase states governed by an alternating Markov renewal process, and by a vector of phase attributes. The phase states describe the state of each relay node (moving or paused) while the phase attributes describe the sojourn time in the current state, the relative location between two consecutive nodes and its movement characteristics: its velocity and direction. Thus, we obtain a process $\mathbf{X}=(\mathbf{P}, \mathbf{A})$ with $\mathbf{P}=\left(P^{i}\right)_{1 \leq i \leq N}$ where $P^{i}$ is the phase process of node $i$, and $\mathbf{A}=\left(\mathbf{E}, \mathbf{M}, \mathbf{L}_{r}\right)$ denotes the joint attribute process, where: $\mathbf{E}=\left(E^{i}\right)_{1 \leq i \leq N}$ denotes the elapsed time since the previous phase transition of node $i, \mathbf{M}=\left(M^{i}\right)_{1 \leq i \leq N}$ denotes the mobility vector of node $i$, if node $i$ is in move phase, and is omitted if node $i$ is in pause phase, and $\mathbf{L}_{r}=\left(L_{r}^{i}\right)_{2 \leq i \leq N}$ denotes the relative location process of node $i$ with respect to node $i-1$. From the definition of $\mathbf{X}$, a state will be denoted by $\mathbf{x}=(\mathbf{p}, \mathbf{a})$ where $\mathbf{a}=\left(\mathbf{e}, \mathbf{m}, \mathbf{l}_{r}\right)$ with the vector $\mathbf{p}=\left(p^{1}, \ldots, p^{N}\right)$ containing the phases of nodes, $\mathbf{e}=\left(e^{1}, \ldots, e^{N}\right)$ the elapsed times of the nodes in their current phases, $\mathbf{m}=\left(\mathrm{m}^{1}, \ldots, \mathrm{m}^{N}\right)$ including the mobility vectors of the nodes, having dimension $N$ when all $p^{j}=1$ and with $m^{j}$ omitted if $p^{j}=$ 0 , and $\mathbf{l}_{r}=\left(l_{r}^{2}, \ldots, l_{r}^{N}\right)$ the relative locations of nodes $2,3, \ldots, N$ relative to nodes $1,2, \ldots, N-1$, respectively.

When the process departs from a state $\mathbf{x}$, the flow of the process describes the deterministic trajectory of $\mathbf{X}$ until the next jump, and is characterized by $\phi(t, \mathbf{x})=$ $\left(\mathbf{p}, \phi_{\mathbf{p}}(t, \mathbf{a})\right)$ with

$$
\phi_{\mathbf{p}}(t, \mathbf{a})=\left(\mathbf{e}+t \mathbf{1}, \mathbf{m}, \mathbf{l}_{r}+t \mathbf{m}_{r}\right), \quad t \in \mathbb{R}
$$

representing the evolution of the component a over time, where $\mathbf{1}$ denotes a vector of $1^{\prime} s$ with dimension $N$ and the vector $\mathbf{m}_{r}=\left(m_{r}^{2}, \ldots, m_{r}^{N}\right)$ contains the relative mobility vectors of nodes $2,3, \ldots, N$ relative to nodes $1,2, \ldots, N-1$, respectively.

For a given phase vector $\mathbf{p}$, denote the set

$$
\left.S_{\mathbf{p}}=\right] 0, \infty\left[^{N} \times S_{M}^{\sum p_{i}} \times S_{L}^{N-1}\right.
$$

where $S_{L}=\left\{x \in \mathbb{R}^{2}:\|x\|<R\right\}$, as the set of the possible values of the attribute process $\mathbf{A}$, that is, the set where all the links of the multihop path are active. Let $\partial S_{\mathbf{p}}$ denote the boundary of the set $S_{\mathbf{p}}, \partial^{-} S_{\mathbf{p}}$ denote the disjoint union of the set of boundary points that take the process into $S_{\mathbf{p}}$, and $B$ denote the set of boundary points at which the multihop path process exits from $S_{\mathbf{p}}$. When the process hits a state in the boundary $B$, which represents the set of states through which the multihop path disconnects, it means that the path breaks and $\mathbf{X}$ jumps to an absorbing state which is denote by $\Delta$. The state space of the joint process $\mathbf{X}$ is denoted by

$$
S_{\mathbf{X}}^{\Delta}=S_{\mathbf{X}} \cup\{\Delta\},
$$

where the set $S_{\mathbf{X}}$ denotes the disjoint union of the sets $S_{\mathbf{p}}^{-}=S_{\mathbf{p}} \cup \partial^{-} S_{\mathbf{p}}$.

For $\mathbf{x} \in S_{\mathbf{X}}$, define $d_{\text {path }}(\mathbf{x})$ as the path duration constrained to no phase transitions of the nodes when departing from state $\mathbf{x}$ (in another words, if the mobile 
nodes maintain their states, is the time to hit a state in $B$, the set of states at which the multihop path is disconnected). This time is equal to infinity if all nodes are in pause phase or all nodes have the same mobility vector.

The function $\lambda: S_{\mathbf{X}}^{\Delta} \rightarrow \mathbb{R}_{0}^{+}$is a measurable function that characterizes the jump rate (or transition rate) from each state of the process. For $\mathbf{x} \in S_{\mathbf{X}}$ the jump rate depends only on the phase and the time since the last phase transition of each node, and is given by the sum of the hazard rate functions $\lambda_{i}(t)=\mathrm{d} F_{i}(t) /\left(1-F_{i}(t)\right)$ of the phase duration distributions $F_{i}, \lambda(\mathbf{x})=\sum_{i=1}^{n} \lambda_{p^{i}}\left(e^{i}\right)$. Since $\lambda(\mathbf{x})$ denotes the rate at which the process will leave from a given state $\mathbf{x}$, and we are considering that the state $\Delta$ (the state of the process when at least one of the links is disconnected) is an absorbing state, $\lambda(\Delta)=0$.

The evolution of $\mathbf{X}$ starting from state $\mathbf{x} \in S_{\mathbf{X}}$ can be constructed as follows. The survival function of the first jump time $T_{1}$ is defined by

$$
G_{\mathbf{x}}(t)= \begin{cases}\exp \left(-\int_{0}^{t} \lambda(\phi(s, \mathbf{x})) \mathrm{d} s\right) & t<d_{\text {path }}(\mathbf{x}) \\ 0 & t \geq d_{\text {path }}(\mathbf{x})\end{cases}
$$

and the state at an instant of time before the first jump is given by the deterministic evolution of the process,

$$
\mathbf{X}(t)=\phi(t, \mathbf{x}), \quad t<T_{1} .
$$

If $T_{1}=d_{\text {path }}(\mathbf{x})$ the path breaks since the process hits a state in $B$ and the next state $\mathbf{X}\left(T_{1}\right)$ is $\Delta$ with probability 1 ; the process then stays in $\Delta$ forever since the jump rate out of $\Delta$ is zero. Otherwise, $T_{1}<d_{\text {path }}(\mathbf{x})$, which means that one of the nodes in the path changes phase and the next state of the multihop process $\mathbf{X}\left(T_{1}\right)$ has distribution $Q\left(\phi\left(T_{1}, \mathbf{x}\right), \cdot\right)$, next defined. The function $Q:\left(S_{\mathbf{X}} \cup B\right) \times \mathscr{E} \rightarrow[0,1]$, is the transition measure where $\mathscr{E}$ denotes the event space of $S_{\mathbf{X}}$, and is such that for $\mathbf{x} \in S_{\mathbf{X}}, Q(\mathbf{x}, \cdot)$ is a probability measure defined by

$$
\begin{array}{ll}
Q\left(\mathbf{x},\left\{\mathbf{x}^{(j)}\right\}\right)=\lambda_{p^{j}}\left(e^{j}\right) / \lambda(\mathbf{x}) ; & p^{j}=1 \\
Q\left(\mathbf{x}, \mathbf{d} \mathbf{x}^{(j)}\right)=\lambda_{p^{j}}\left(e^{j}\right) / \lambda(\mathbf{x}) F_{M}(\mathrm{~d} m) ; & p^{j}=0
\end{array}
$$

where the new state of the process, $\mathbf{x}^{(j)}$, is next defined, and for $\mathbf{x} \in B$ we have $Q(\mathbf{x},\{\Delta\})=1$. The interpretation of $\mathbf{P}$ and $\mathbf{A}$ makes it clear that from any $\mathbf{x}=$ $(\mathbf{p}, \mathbf{a}) \in S_{\mathbf{X}}$ it is only possible to jump to a state where a node changes its phase characteristics (phase, elapsed time in the phase, and mobility vector) and all the other values of the components remain the same. Thus, when $p^{j}=1, \mathbf{x}^{(j)}$ coincides with $\mathbf{x}$ except for the fact that the phase of node $j$ is a pause phase, $p^{j}=0$, and the corresponding elapsed time in the phase is null, $e^{j}=0$ (and its mobility vector will be omitted). Conversely, when $p^{j}=0$, the new state of the process, $\mathbf{x}^{(j)}$, coincides with $\mathbf{x}$ except on the part concerning to node $j$ whose phase becomes a move phase, $p^{j}=1$, the corresponding elapsed time in the phase is null, $e^{j}=0$, and the mobility vector is $m$. When the new state of the process is chosen, the process restarts from $\mathbf{X}\left(T_{1}\right)$ in a similar way. For a detailed description of the multihop path model, please see [1]. 


\section{Mean path duration}

Given the state of the multihop path process $\mathbf{x} \in S_{\mathbf{X}}^{\Delta}$, the mean path duration is denoted by

$$
D(\mathbf{x})=\mathbb{E}_{\mathbf{x}}\left(\int_{0}^{\infty} \mathbf{I}_{S_{\mathbf{X}}}(\mathbf{X}(s)) \mathrm{d} s\right)
$$

where $\mathbf{I}_{A}$ is the indicator function of a set $A$. Note that since $\mathbf{I}_{S_{\mathbf{X}}}(\mathbf{X}(s))$ denotes the indicator function that the process $\mathbf{X}$ belonging to a state where all the links of the multihop path are connected, the integral in (3) denotes the amount of time that the process $\mathbf{X}$ remains connected, when departing from a state where all links are connected. Thus, the expected value, $D(\mathbf{x})$, is in fact the mean path duration of the multihop path process.

In [1] it is proved that the mean path duration written as the expectation of a functional of the multihop path process is the unique solution of a system of integrodifferential equations. However, any direct method to solve them is quite problematic and depends very much on the specific characterization of the multihop path process (number of nodes, deterministic motion, jump rate, transition measure). To provide a way to calculate numerically $D(\mathbf{x})$ a recursive scheme is proposed for a feasible computation of the mean path duration.

Let $D^{0}$ be a function such that $D^{0}(\mathbf{x})=0$ for all $\mathbf{x} \in S_{\mathbf{X}}^{\Delta}$ and let $\mathscr{O}$ be an operator mapping the set of bounded measurable functions on $S_{\mathbf{X}}^{\Delta} \cup B$ into itself. The action of the operator $\mathscr{O}$ on $D^{0}$ originates the function $D^{1} \equiv \mathscr{O} D^{0}$ defined by

$$
D^{1}(\mathbf{x})=\mathbb{E}_{\mathbf{X}}\left(\int_{0}^{T_{1}} \mathbf{I}_{S_{\mathbf{X}}}(X(s)) \mathrm{d} s+D^{0}\left(\mathbf{X}\left(T_{1}\right)\right)\right), \mathbf{x} \in S_{\mathbf{X}}^{\Delta} .
$$

Iterating $k(\geq 1)$ times the operator $\mathscr{O}$ on $D^{0}$ results into the function $D^{k} \equiv \mathscr{O}^{k} D^{0}$ given by

$$
D^{k}(\mathbf{x})=\mathscr{O} D^{k-1}(\mathbf{x})=\mathbb{E}_{\mathbf{x}}\left(\int_{0}^{T_{1}} \mathbf{I}_{S_{\mathbf{X}}}(X(s)) \mathrm{d} s+D^{k-1}\left(\mathbf{X}\left(T_{1}\right)\right)\right)
$$

for $\mathbf{x} \in S_{\mathbf{X}}^{\Delta}$.

The equation above signifies that, if the state of the process after $k-1$ phase transitions is known, the state of the process after $k$ phase transitions is just given by the evolution of the process until the next phase change. The metric $D^{k}(\mathbf{x})$ denotes the mean path duration constrained to at most $k$ jumps of the multihop process $\mathbf{X}$, when departing from state $\mathbf{x}$. As the number of jumps increases, in the limit, the mean path duration $D(\mathbf{x})$ defined by (3), is obtained

$$
\lim _{k \rightarrow \infty} D^{k}(\mathbf{x})=D(\mathbf{x})
$$

Let $f: S_{\mathbf{X}}^{\Delta} \cup B \rightarrow \mathbb{R}^{+}$be a bounded measurable function and for $\mathbf{x} \in B$ define $f(\mathbf{x}) \equiv \lim _{t \downarrow} f(\phi(-t, \mathbf{x}))$. Denote the expected value of $\mathbf{X}$ just after a jump from $\mathbf{x}$ by 


$$
\mathscr{Q} f(\mathbf{x})=\int_{S_{\mathbf{X}}^{\Lambda}} f(\mathbf{y}) Q(\mathbf{x}, \mathbf{d y}),
$$

where for $\mathbf{x} \in B, \mathscr{Q} f(\mathbf{x})=f(\Delta)$.

For $\mathbf{x} \in S_{\mathbf{X}}$, the state of $\mathbf{X}$ after a short time $t$ is, roughly, $\phi(t, \mathbf{x})$ with probability $1-\lambda(\mathbf{x}) t+o(t)$, while with probability $\lambda(\mathbf{x}) t+o(t)$ the process jumps to another state $\mathbf{X}(t)$ with transition measure $Q$ and all other events have probability $o(t)$. Thus, we have

so that

$$
\mathbb{E}_{\mathbf{x}}(f(\mathbf{X}(t)))=(1-\lambda(\mathbf{x}) t) f(\phi(t, \mathbf{x}))+\lambda(\mathbf{x}) t \mathscr{Q} f(\phi(t, \mathbf{x}))+o(t),
$$

$\frac{1}{t} \mathbb{E}_{\mathbf{x}}(f(\mathbf{X}(t))-f(\mathbf{x}))=\frac{1}{t}(f(\phi(t, \mathbf{x}))-f(\mathbf{x}))+\lambda(\mathbf{x})(\mathscr{Q} f(\phi(t, \mathbf{x}))-f(\phi(t, \mathbf{x})))+o(1)$.

Denote by $\mathscr{A} f(\mathbf{x})$ the derivative of equation (5) where, in order to define the derivative of $f$ with respect to the flow $\phi(t, \mathbf{x})$ in a rigorous way, we need to define its phase function $f_{\mathbf{p}}: S_{\mathbf{p}}^{-} \rightarrow \mathbb{R}^{+}$by $f_{\mathbf{p}}(\mathbf{a})=f(\mathbf{x})$. Therefore, as $t \rightarrow 0$ in equation (6) we obtain

$$
\mathscr{A} f(\mathbf{x})=\mathscr{V} f(\mathbf{x})+\lambda(\mathbf{x})(\mathscr{Q} f(\mathbf{x})-f(\mathbf{x})),
$$

for $\mathbf{x} \in S_{\mathbf{X}}$, where to simplify the notation we write $\mathscr{V} f(\mathbf{x})$ instead of the more accurate $\mathscr{V}_{\mathbf{p}} f_{\mathbf{p}}(\mathbf{a})$. Also any reference to a function $t \rightarrow f(\phi(t, \mathbf{x}))$ should be read as $t \rightarrow f_{\mathbf{p}}\left(\phi_{\mathbf{p}}(t, \mathbf{a})\right)$. The function $\mathscr{V}_{\mathbf{p}}$ is the vector field and $\phi_{\mathbf{p}}(t, \mathbf{a})$ is the unique integral curve of $\mathscr{V}_{\mathbf{p}}$ such that for almost all $t$,

$$
\frac{\mathrm{d}}{\mathrm{d} t} f_{\mathbf{p}}\left(\phi_{\mathbf{p}}(t, \mathbf{a})\right)=\mathscr{V} / \mathbf{p} f_{\mathbf{p}}\left(\phi_{\mathbf{p}}(t, \mathbf{a})\right), \quad \phi_{\mathbf{p}}(0, \mathbf{a})=\mathbf{a}
$$

is satisfied, since the function $t \rightarrow f_{\mathbf{p}}\left(\phi_{\mathbf{p}}(t, \mathbf{a})\right)$ is differentiable almost everywhere on $\left[0, d_{\text {path }}(\mathbf{x})[\right.$.

The next result follows from theorems 32.2 and 32.10 of Davis [3] conveniently applied to the expectation functional $D^{k}$ of the PDMP $\mathbf{X}$ with finite time horizon and taking into account the specific boundary conditions.

Proposition 1. Suppose that the function $D^{k-1}$ is given. For each $\mathbf{x} \in S_{\mathbf{X}}, t \rightarrow$ $D^{k}(\phi(t, \mathbf{x}))$ is an absolutely continuous function on $\left[0, d(\mathbf{x})\left[\right.\right.$ and $D^{k}$ is the unique bounded solution of the equations

$$
\mathscr{V} f(\mathbf{x})+\lambda(\mathbf{x})\left(\mathscr{Q} D^{k-1}(\mathbf{x})-f(\mathbf{x})\right)=-1, \quad \mathbf{x} \in \mathbf{S}_{\mathbf{X}},
$$

and at a boundary state $\mathbf{x} \in B, f(\mathbf{x})=f(\Delta)=0$.

Note that in equation (7) the operator $\mathscr{Q}$ acts only on the given function $D^{k-1}$, so that the respective equations are ODEs. Combining this result with (4) provides a recursive way for computing the mean path duration $D$. For a complete proof of the results presented in this Section, please see [5]. 
Computing $D^{k}$ requires only to solve first order ordinary differential equations. The results of these calculations are then used to compute the next iteration $k+1$. Since they are independent ODEs they can be computed using parallel computation. The convergence of the solution depends on how large $k$ has to be before $D^{k}$ is close to $D$. Any direct implementation of these equations requires a discretization of the state space and solving at each grid point an independent ODE, providing the data for calculating the next iteration. It is unrealistic to hope that numerical solutions are possible for a medium size number of links in a single workstation due to the great number of computer processing cycles and the need of storing large amounts of data. However, it is possible to solve the equations in a single workstation in the case of one or two links and in one dimensional ad hoc networks.

\section{Numerical Results}

In this section we illustrate an application of the preceding results and study the effect of the independence link assumption. The scenario proposed intends to model a scenario where nodes move with relatively low velocities and pause times. We consider that the phase durations are exponentially distributed with means of $30 \mathrm{~s}$ and $60 \mathrm{~s}$ in move and pause phase, respectively. The transmission range of a node is set up to $250 \mathrm{~m}$. The mobility vector is obtained choosing a velocity $(\mathrm{m} / \mathrm{s})$ and direction of nodes uniformly distributed in $] 1,20[$ and $] 0,2 \pi[$, respectively. For a multihop path with $N$ nodes, initially each node $i(2 \leq i \leq N)$ is deployed inside node $i-1$ 's radio coverage with an angle uniformly distributed in $] 0,2 \pi[$ and with a distance following a triangular distribution in the interval $(0,250)$ with mode 62.5 . The initial phase of a node is picked randomly with probabilities proportional to the mean time spent in the phase.

Figure 1 shows the results of the mean path duration after each iteration for different link count. The departure states of the multihop path were sampled according to the initial distribution and their respective mean path durations were estimated in each iteration using Monte Carlo methods (in a single workstation). The results were averaged out in the final of each iteration. The difference between iterations gets smaller as the number of links increases since it gets more likely that a path failure occurs after a small number of phase transitions. However, all curves have converged before iteration 25 .

In Figure 2 we investigate the impact of neglecting the dependency between links in the mean path duration and study the impact of the mean time in pause phase on the mean path duration. Numerical routines were developed for independent links. We conclude that the mean path duration increases with the increase of the mean time in pause phase, and the independent link assumption leads always to an underestimation of the mean path duration. The observed percentage errors from assuming independent links were always higher than $3.5 \%$ and achieved values higher than $25 \%$. The results are rather sensitive to the mean time in pause phase and getting an estimate for the mean path duration using the link independence assumption 


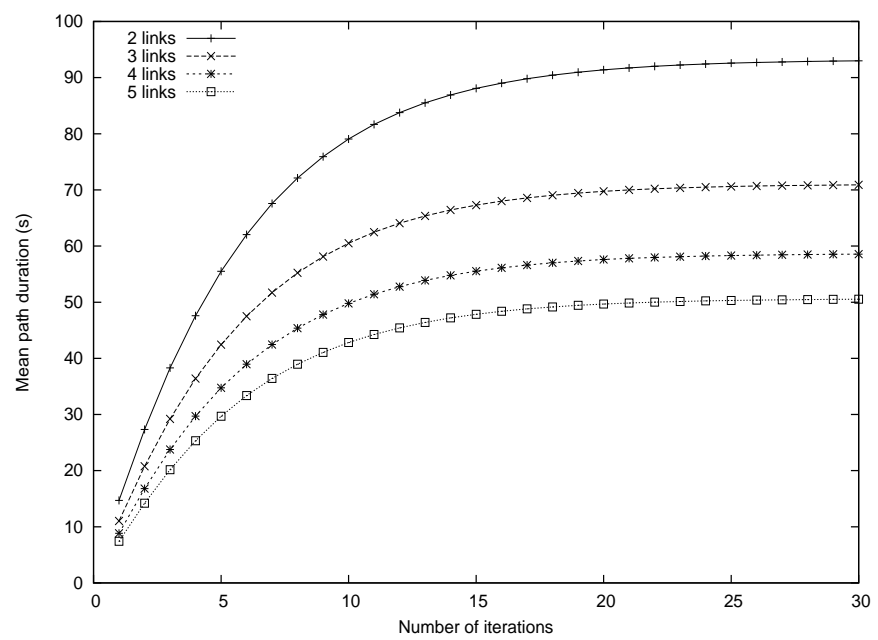

Fig. 1 Mean path duration after each iteration.

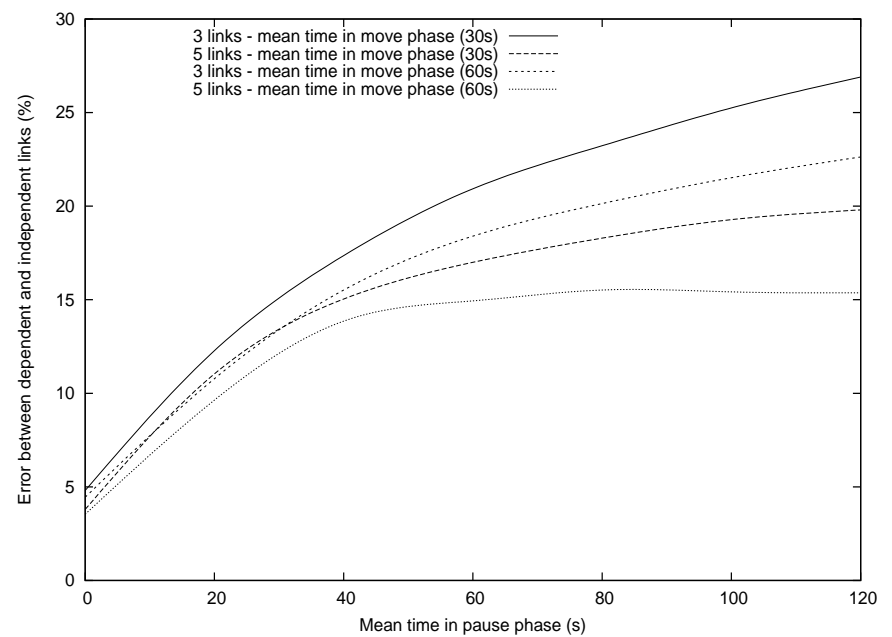

Fig. 2 Error (\%) between mean path duration using dependent and independent links vs mean time in pause phase.

may in fact lead to a large bias when the inactive time of a node is large. Smaller values of the percentage errors occur with paths with a higher number of hops and in scenarios where mobile nodes have higher mobility (no pause phase).

Figure 3 shows the impact of the mean time in move phase on the mean path duration. We conclude that with the increase of the mean time in move phase, the mean path duration decreases and also the percentage error arising from assuming independent links. We can again observe that higher percentage errors occur in sce- 


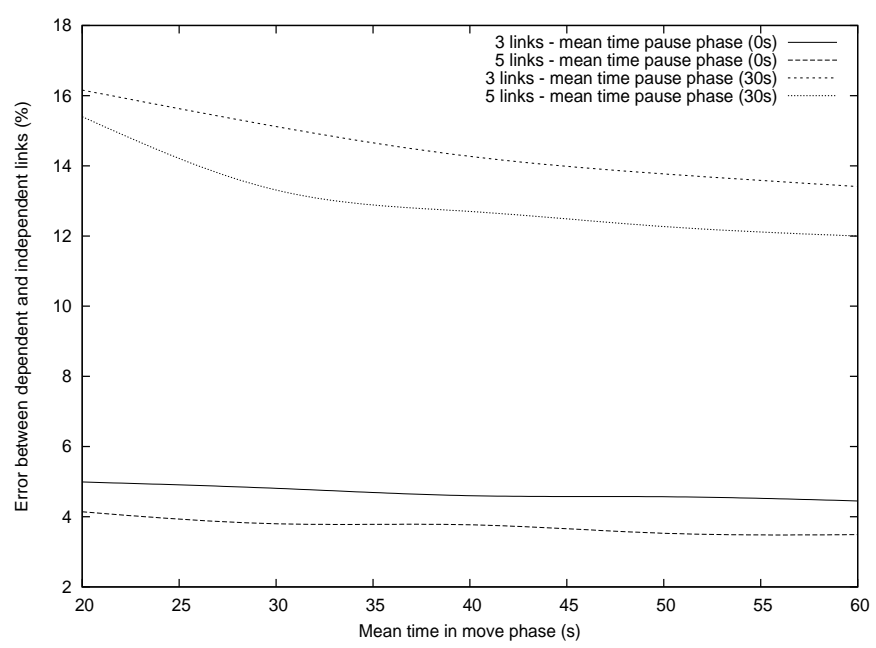

Fig. 3 Error (\%) between mean path duration using dependent and independent links $v s$ mean time in move phase.

narios where nodes have low mobility (inclusion of pause phases) and in multihop paths with a small number of hops.

In Figure 4 we observe the impact of the mobile nodes velocity on the mean path duration. We conclude that with the increase of the mean node velocity, leading to a decrease of the mean path duration, the percentage error by assuming independent links increases, so higher node velocity associated with low node mobility (long pause phases) increases the underestimation error of mean path durations when the independent links assumption is used.

\section{Conclusion}

This paper uses an analytical framework to characterize the random behaviour of a multihop path under a PDMP that allows to describe the mean path duration through a set of ordinary differential equations and a recursive scheme for its computation. The results obtained using this model were compared with the corresponding results obtained assuming that links behave independently. We concluded that in scenarios with a small number of links, high velocities, and low node mobility (inclusion of long pause phases) the independent links assumption can lead to large underestimation of the mean path duration. In these scenarios, the percentage error can achieve values higher than $25 \%$. In the best case scenario, that is, with no pause phases, low velocities and high number of links, the observed percentage errors were always higher than 3.0\%. These results can be used to improve the performance of routing algorithms in MANETs. 


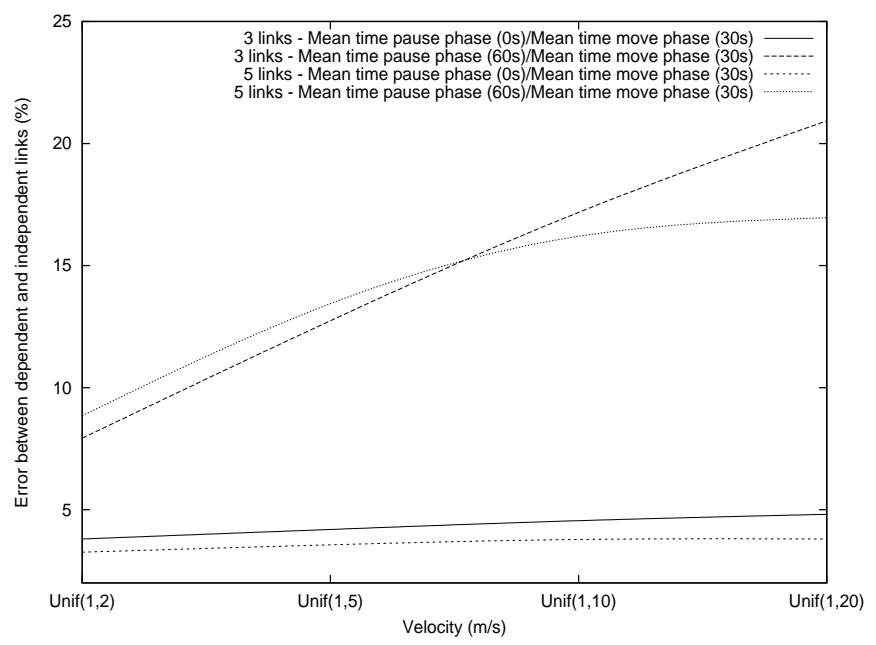

Fig. 4 Error (\%) between mean path duration using dependent and independent links $v s$ mobile node velocity.

\section{References}

1. Antunes N, Jacinto G, and Pacheco A (2010) An analytical framework to infer multihop path reliability in MANETs. SIGMETRICS '10: Proceedings of the ACM SIGMETRICS International Conference on Measurement and Modeling of Computer Systems 323-332, New York, USA.

2. Bai F, Sadagopan N, Krishnamachari B, and Helmy (2004) A Modeling path duration distributions in MANETs and their impact on reactive routing protocols. IEEE J. Select. Areas Commun 22(7):1357-1373.

3. Davis M (1993) Markov Models and Optimization. Chapman and Hall, London/New York.

4. Han Y, La RJ, Makowski AM, and Lee S (2006) Distribution of path durations in mobile ad-hoc networks: Palm's theorem to the rescue. Computer Networks 50(12):1887-1900.

5. G. Jacinto (2011) Modeling and performance evaluation of mobile ad hoc networks. PhD thesis, Instituto Superior Técnico, Technical University of Lisbon, Lisbon, Portugal.

6. La RJ and Han Y (2007) Distribution of path durations in mobile ad hoc networks and path selection. IEEE/ACM Trans. Netw. 15(5):993-1006.

7. McDonald AB and Znati T (1999) A path availability model for wireless ad-hoc networks. Proc. IEEE Wirel. Commun. Netw. Conf. 1:35-40, Orleans, LA, USA.

8. Xu S, Blackmore KL, and Jones HM (2007) An analysis framework for mobility metrics in mobile ad hoc networks. EURASIP J. Wirel. Commun. and Network. 1(16):26-26. 